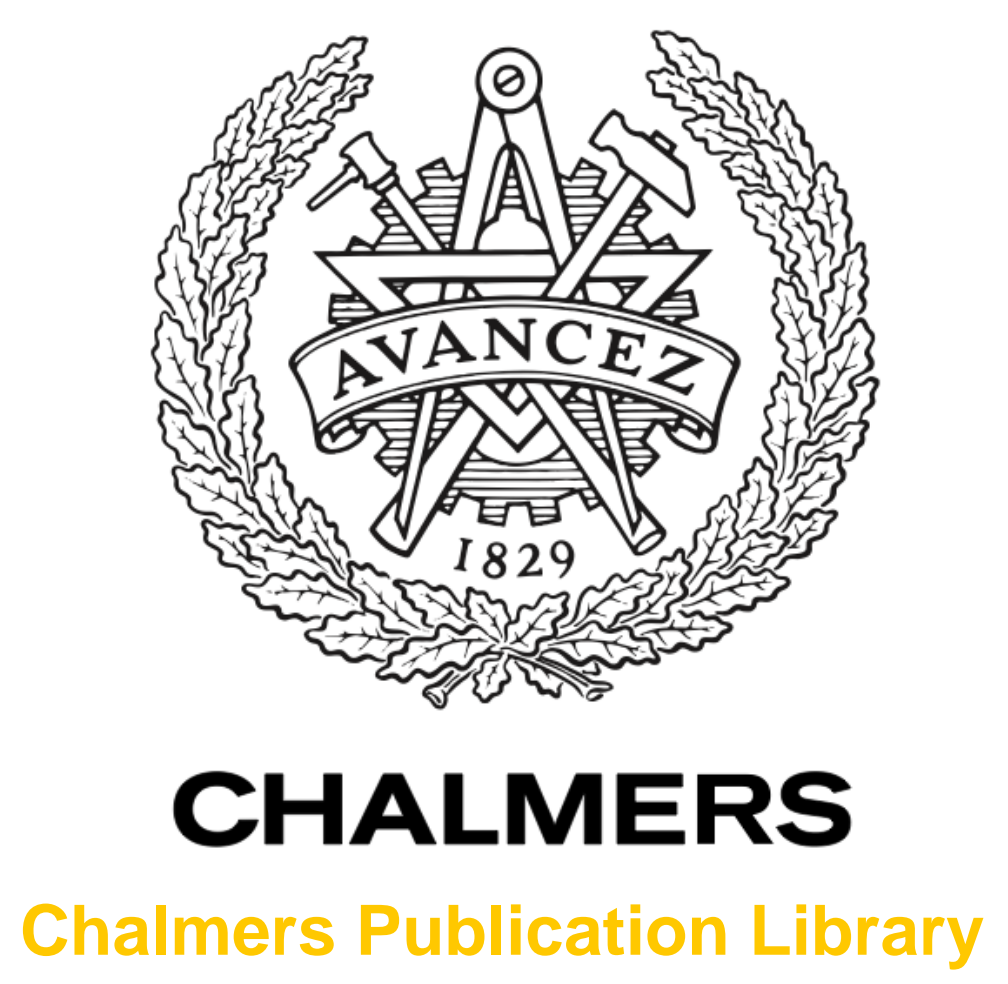

\title{
Gap Waveguide PMC Packaging for Improved Isolation of Circuit Components in High-Frequency Microwave Modules
}

This document has been downloaded from Chalmers Publication Library $(\mathrm{CPL})$. It is the author's version of a work that was accepted for publication in:

IEEE Transactions on Components, Packaging, and Manufacturing Technology (ISSN:

2156-3950)

Citation for the published paper:

Zaman, A. ; Alexanderson, M. ; Vukusic, T. (2014) "Gap Waveguide PMC Packaging for Improved Isolation of Circuit Components in High-Frequency Microwave Modules". IEEE Transactions on Components, Packaging, and Manufacturing Technology, vol. 4(1), pp. 1625 .

http://dx.doi.org/10.1109/tcpmt.2013.2271651

Downloaded from: http://publications.lib.chalmers.se/publication/193539

Notice: Changes introduced as a result of publishing processes such as copy-editing and formatting may not be reflected in this document. For a definitive version of this work, please refer to the published source. Please note that access to the published version might require a subscription. 


\title{
Gap Waveguide PMC Packaging For Improved Isolation of Circuit Components in High Frequency Microwave Modules
}

\author{
Ashraf Uz Zaman, Mats Alexanderson, Member, IEEE, Tin Vukusic, Per-Simon Kildal, Fellow, IEEE
}

\begin{abstract}
In this work, Perfect Magnetic Conductor (PMC) based packaging technique was used to improve the isolation performance among various microwave circuit components such as high gain amplifier chains. In this approach, a periodic structure (such as metal pin rows) together with the ground plane of the substrate, created a stopband for unwanted parallel-plate or cavity modes as well as substrate modes, and thereby suppressed problems of circuit resonances and related package phenomena. This paper describes two Ka-band amplifier chains that were tested with this new packaging technique. Firstly, a single amplifier chain was tested for maximum stable gain operation, and it was found that stable gain of more than $65 \mathrm{~dB}$ was achieved whereas traditional metal wall package with RF absorber offered stable gain of $40 \mathrm{~dB}$, thus showing significant isolation improvement. Secondly, two high gain amplifier chains were placed side by side and their mutual isolation was tested. With proposed gap waveguide packaging, a minimum isolation of $78 \mathrm{~dB}$ was achieved whereas a complete metal shield provided a minimum isolation of only $64 \mathrm{~dB}$ over the band of interest.
\end{abstract}

Index Terms-Isolation, PMC packaging, parallel-plate modes, substrate modes, stable gain, self-oscillation.

\section{INTRODUCTION}

$\mathrm{T}$ HE technology of millimeter wave circuits has been developing for many years and is now becoming mature enough to be used in realization of complex assemblies and microwave modules. Historically, the first millimeter wave applications have been for military and space purposes with customized microwave modules. However, with the emergence of new civilian applications, such as automotive sensors, high speed wireless LANS, microcell communication, point-to-point links and point-to-multipoint links, tracking systems etc. cost and mass manufacturability are becoming predominant factors.

Most of mm-wave microwave systems have to operate at outdoor, under harsh weather conditions and therefore have to

Manuscript received September, 2012. This work has been supported in part by the Swedish Research Council VR, and by The Swedish Governmental Agency for Innovation Systems (VINNOVA) within the VINN Excellence Center Chase.

A.Uz Zaman and P.-S. Kildal are with the Department of Signals and Systems, Chalmers University of Technology, SE-412 96 Göteborg, Sweden.

phone: +46 317721794 ; fax: +46 3177217 48; e-mail: (zaman@ chalmers.se; per-simon.kildal@chalmers.se)

M. Alexanderson and T. Vukusic are with R\&D section of Trebax AB, SE42131 Västra Frölunda, Göteborg, Sweden (e-mail: mats@trebax.se; tin@trebax.se). be shielded, i.e., packaged in a proper way. Packaging of mmwave integrated hybrid circuits (MICs), monolithic integrated circuits (MMICs) and micro-electromechanical systems (MEMSs) have to provide protection against mechanical stress and environmental condition. Also, to comply with the smaller size requirements and compactness for mm-wave microwave modules, large amount of electronic components are to be placed into a confined area. For such high density microwave modules, RF packaging is more and more important in terms of isolation and interference suppression. If not properly controlled, the interference or cross-talk can cause each system to malfunction and even fail. Thus one of the most challenging design aspects of mm-wave RF packaging is to maintain high isolation among the adjacent microwave circuit components and suppress unwanted RF interference [1]-[3].

In a common microwave module, circuit components such as MMICS are placed on a dielectric substrate which can carry the necessary interconnect lines, the passive components as shown in Fig.1. Enclosing the complete circuitry in a metal package usually degrades the RF circuit performance because of the onset of package resonances and more, in general, due to the interactions of electromagnetic fields (produced by the signal propagating in the electronic circuitry) with the package itself.

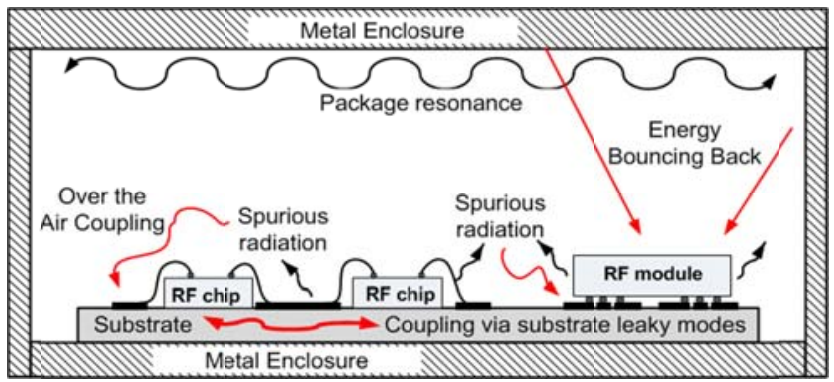

Fig.1 Packaged microwave module with various undesired coupling phenomena

The MMICs, hybrid circuits, other passive components or interconnect lines may be based on different types of transmission line media such as microstrip lines or coplanar waveguides (CPWs). Published studies in [4]-[6] show that significant power leakage exists on various printed circuit transmission lines, often related to surface waves in the dielectric substrate causing serious cross-talk and interference problems. Particularly in the case of top-covered microstrip line, this leakage begins at a much lower frequency than 
expected and become a matter of serious concern for power loss as well as cross-talk issue [5]. Similarly, conventional packaging of $\mathrm{CPW}$ modifies the $\mathrm{CPW}$ into a conductorbacked CPW (CBCPW), and this thereby generates power leakage in the form of coupling to parasitic parallel-plate (PP) modes [6], [7]. Typically, electrically large CBCPWs will support a dense (almost continuous) spectrum of PP modes and thereby cause significant radiation through these modes at their resonance frequencies. Apart from this problem of PP mode leakage and radiation, at mm-wave frequencies, interconnects and transitions between different components or even the interconnecting signal lines may produce spurious radiation or standing waves. This can easily get coupled (over the air) to the neighboring circuit elements resulting in interference and cross-talk. Fig. 1 shows some of the possible ways of electromagnetic field coupling and unwanted energy leakage between the adjacent circuit elements.

A preventive solution to provide good isolation between two neighboring circuit components is the multi-compartment microwave modules shown in Fig.2. This solution consists of using a separate metallic shielding enclosure for each critical component (typically the active components). Usually, such metallic enclosures will be partly filled with lossy microwave absorbers to dampen the high Q cavity modes [8], [9]. Also, there is an absolute need for adding vias near the "hot spots" to stop energy coupling via the substrate leaky waves [10][12]. There are also other more complex ways of suppressing the substrate leaky modes, such as the insertion of a doped silicon ( $\mathrm{Si}$ ) sub-mount layer (high resistivity layer) in the substrate [7], tailoring of the substrate thickness to stop the power coupling to the $T M_{0}$ surface mode, or using guard rings or deep trench isolation techniques [13],[14]. Also, with the advent of High- impedance electromagnetic surfaces [15], some researchers have used this high impedance surface as a mean of suppressing parallel-plate noise in high speed circuits [16], [17]. Unlike traditional localized noise-suppression methods, these techniques offer the ability to suppress the noise coupling via the PP waveguide modes over the entire substrate. In practice, the efficiency of the different techniques available for reducing cross-talk goes down with frequency; the higher the frequency, the lower is usually the effectiveness of the techniques mentioned so far.

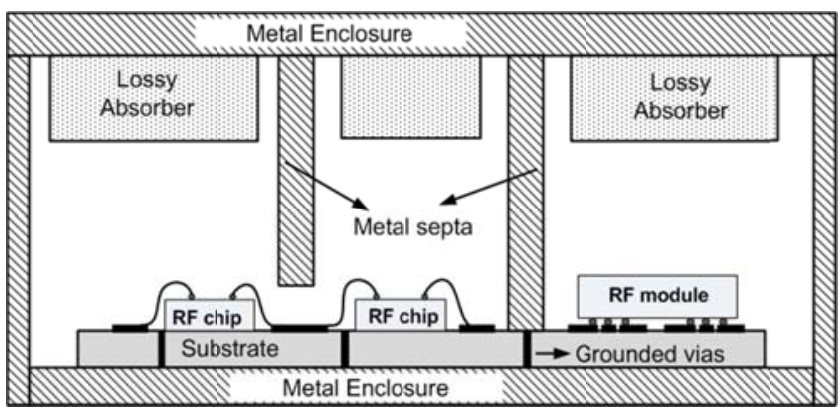

Fig.2 Traditional multi-compartment microwave module

In this paper, we propose a new packaging technique which deals with cavity resonances within the metal enclosure, over the air coupling between the adjacent components, as well as the coupling via the substrate modes, thereby improving the isolation among the circuit components. This new technique is easily scalable to high frequency and shows consistent performance over different microwave frequency bands [18]. The proposed new packaging technique is shown in Fig.3. It is an extension of the recently developed gap waveguide technology. This technology uses the basic cut-off of a PECPMC parallel-plate waveguide configuration to control the electromagnetic wave propagation between the two parallel plates. As PMCs are not available in nature, the PMC condition must be emulated by artificial magnetic conductor (AMC) in the form of periodic structures such as metal pins [19] or mushroom structures [15]. All the global parallel-plate (PP) modes are in cutoff within the frequency band where the AMC has high enough surface impedance to create a stopband of the PP modes and thereby allows only the desired waves along ridges or grooves to propagate within the gap waveguide structure. The concept of gap waveguide technology was first published in [20], [21] and later ridge gap waveguide concept was experimentally demonstrated in [22], [23]. Also the existence of several well-defined Quasi-TEM modes in the single parallel-plate hard waveguide was presented in [24]. Numerical studies on designing different artificial magnetic surface (AMC) for a particular frequency band were also studied thoroughly in [25]. Using the gap waveguide concept and periodic metal pin surface as AMC, microwave components such as high $\mathrm{Q}$ bandpass filters, wideband slot array antennas, rat race hybrids, phased array antennas, etc. were designed [26]-[29]. For the packaging application, the ground of the circuit board is ideally the Perfect Electric Conductor (PEC), and the lid should work as a Perfectly Magnetic Conductor (PMC) creating a PEC-PMC parallelplate structure where all unwanted parallel-plate modes are suppressed. The application for packaging was first demonstrated successfully for a passive microstrip line in [30]. Later it was applied to a multiport antenna feed network in [31] and to improve the performance of well-known coupled line microstrip filter [32]. In all these cases, it was observed that unwanted cavity modes and surface waves were efficiently suppressed within the band of interest.
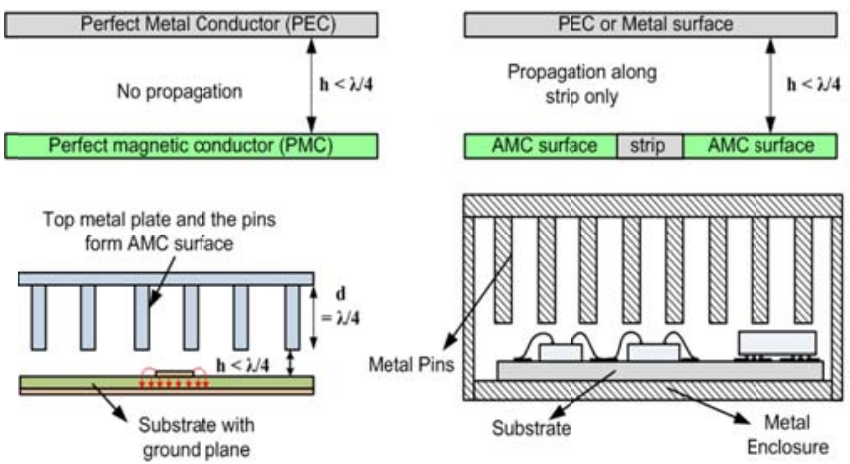

Fig.3 New PMC gap waveguide packaging concept.

The PMC packaging technique is also a way to include packaging from the start when designing microwave circuits, instead of struggling with it afterwards. This saves computer time and therefore, also reduces development time and is referred to as numerical PMC prepackaging [33]. 
In the present work, we used and evaluated the PMC packaging technique on a Ka-band high gain amplifier chain having four cascaded units with an automatic gain control option. First, we varied the gain of each amplifier unit and looked for the oscillation tendency of the chain when the total gain was high enough. The self-resonance test was of particular interest in this case. Secondly, we had put two amplifier chains side by side, but running in opposite direction, and determined the improvement of the isolation between them by using the PMC packaging technique. In either the cases, the proposed PMC or gap waveguide packaging technique were compared with conventional metal cavities with RF absorber and via based packaging.

\section{FEATURES AND DESIGN OF GAP WAVEGUIDE PACKAGE APPLIED TO AN AMPLIFIER CHAIN}

Provided the biasing circuit is properly designed, and leakage through the bias lines is suppressed, instability in a millimeter-wave amplifier is mainly the result of unwanted feedback across the amplifier and such feedback is normally introduced during the packaging [11], [34]-[36]. This can be avoided if the chip size is so small that the metal cavity (inside which it is packed) has no resonances within the frequencies of interest. However, practical semiconductor chips for mmwave frequencies are normally larger than this. Also, if someone wants to build up a transmit/receive module based on commercially packaged SMT chips, i.e. QFN style packages, the chip size is typically pre-defined with dimension of $3 \times 3 \times 1 \mathrm{~mm}^{3}$ or $5 \times 5 \times 1 \mathrm{~mm}^{3}$. Therefore, engineers are left with options such as placing lossy absorber material in the packaged cavity or relocating a particular circuit element to a different position in the cavity, in order to solve problems with cavity resonances and other package phenomena. These particular approaches are effective if the gain in each cavity is not very high, typically below $20 \sim 22 \mathrm{~dB}$, and they become less effective if larger gains are required of the amplifiers in every cavity. Also, these traditional techniques cause additional production costs and delay in production cycle. Thus, it is important to have a more robust packaging solution.

To deal with the above-mentioned issues, we propose a new packaging technique for high frequency multi compartment microwave modules that has following good features:

- It works within a desired frequency band and is totally independent of cavity dimension.

- It is easily scalable to different frequency bands.

- It is very efficient in terms of suppressing both coupling over the air and coupling via the substrate modes within a large frequency band of interest.

- It suppresses the cavity modes within the desired band.

- It improves overall insertion loss of complete microwave circuit as leakages to unwanted modes are suppressed.

- It reduces the need for trial and error process with absorber placements for removing the cavity modes after the unpackaged design has been completed.
- It removes the need for good metal contact between the circuit board and the metal enclosure, or in other words; there is no need for proper grounding and the conductive adhesive or gasket material used for multi-compartment microwave modules.

- Running signal lines from one compartment to another compartment within the microwave module is also done without degrading the isolation performance.

- The isolation performance depends no more on mechanical issues such as stress and corrosion, joint reliability, surface properties, and deformations and apertures caused by thermal expansions or contractions.

Instead of providing shielding by using a smooth metal wall, this new packaging technique requires a pre-designed artificial PMC surface, placed on top of the microwave circuitry at a distance less than quarter wavelength from the ground plane at the highest frequency of interest. This PMC surface can be any periodic structure such as a mushroom type electromagnetic bandgap (EBG) surface [15], metal pins known as a bed of nails [19], and a bed of springs [37]. In this section, we describe the design of a gap waveguide package for an amplifier chain having four cascaded amplifiers.

A full-wave numerical simulation tool is needed in order to successfully design a packaging module for active highfrequency circuits in order to include all the electromagnetic effects related to coupling to substrate modes and cavity resonances and coupling from one active circuit to others via such modes and resonances [38]. In the present work, we modeled a single amplifier chip as a square bulk material block with dimensions $3 \times 3 \times 1 \mathrm{~mm}^{3}$ (similar to the size of the amplifier chips), and we placed this block on the substrate. The ceramic block also included a grounded GaAs substrate of thickness $0.1 \mathrm{~mm}$ and $\mathrm{I} / \mathrm{O}$ lines with $50 \Omega$ microstrip lines. The main substrate used in this work was an Arlon CLTE-AT substrate with a thickness of $0.254 \mathrm{~mm}$, and the substrate size was $30 \times 25 \mathrm{~mm}^{2}$. The biasing circuitry and bias traces were not included in this model to simplify the simulations. The main objective here was to check the suppression of both the cavity modes, and the external coupling over-the-air from one element to another, particularly in the presence of the main substrate. This numerical model is illustrated in Fig.4.

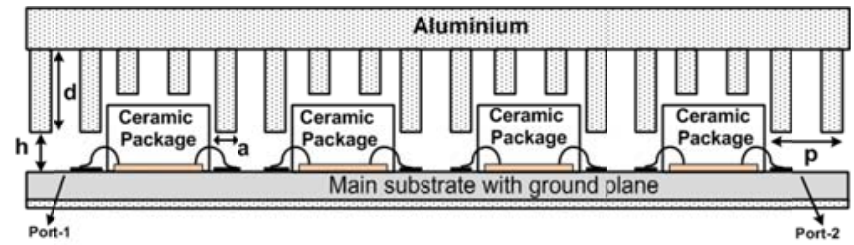

Fig.4 Gap waveguide packaging model for full-wave simulation; $\mathrm{a}=0.8 \mathrm{~mm}$, $\mathrm{h}=0.5 \mathrm{~mm}, \mathrm{~d}=2.25 \mathrm{~mm}, \mathrm{p}=2.25 \mathrm{~mm}$.

For convenience of manufacturing, in the present work we chose periodic metal pins as an artificial PMC, designed to work at Ka band. This pin design started with the unit cell to ensure a stopband for parallel-plate modes within the band of 
interest. To do so, the design curves given in [25] were used. For the pin dimensions given in Fig.5, the stopband ranges from $26 \mathrm{GHz}$ to $46 \mathrm{GHz}$. The corresponding dispersion diagram is shown in Fig. 5.

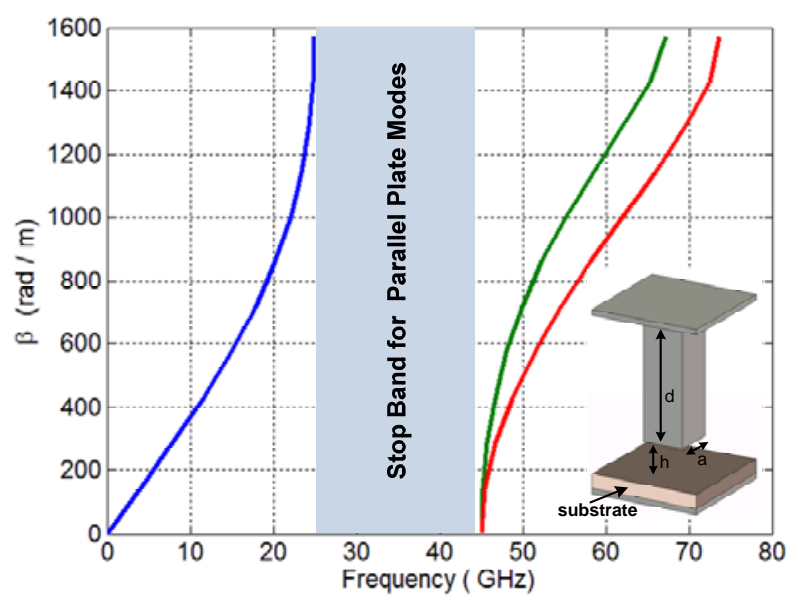

Fig. 5 Dispersion diagram for unit cell; $\mathrm{a}=0.8 \mathrm{~mm}, \mathrm{~h}=0.5 \mathrm{~mm}, \mathrm{~d}=2.25 \mathrm{~mm}$.

As mentioned in [25], the air gap spacing ' $h$ ' between the substrate and the pin plays a very significant role in determining the extent of the stopband. The smaller the value of this air gap, the larger is the stopband for parallel plate modes. In this work we had chosen a value of $0.5 \mathrm{~mm}$ for the parameter ' $h$ ' everywhere in the package except at the location of the amplifier chips. Regular surface mount amplifier chips usually have a height of approximately $0.8 \mathrm{~mm}$. This led to the shortening of the pin heights above the amplifier location by $0.8 \mathrm{~mm}$ and consequently the value of ' $h$ ' was changed in these locations. This would create a lid with non-uniform pin heights, breaking the homogeneity in the periodic structure and affecting the stopband for parallel-plate modes as well the isolation performance. It is to be noted that we tried to place two rows of pins in the space between the two dummy amplifier chips, so as to provide enough isolation between the two adjacent elements on the main substrate.

In reference [20] and [26] it was shown that with two rows of pins the electromagnetic wave propagation was suppressed up to a level of $45 \mathrm{~dB}$. This was considered to be enough for isolation between adjacent two elements in an amplifier chain, because each of these amplifiers was expected to operate with a maximum gain of approximately $18 \mathrm{~dB}$. The expected overall isolation between the input and output ports was of course much better than $45 \mathrm{~dB}$ because there were in total 8 pin rows between these two points.

The computed isolation between two cascaded dummy MMICs with two rows of pins between them is shown in Fig. 6(a), for the ideal PMC packaging case (located at height $1.0 \mathrm{~mm}$ above $\mathrm{PCB}$ ), for the case of a uniform pin grid at height $0.5 \mathrm{~mm}$ above the $\mathrm{PCB}$, and actual non-uniform height pin lid. The total isolation between the input and output ports of the whole chain is shown in Fig. 6(b). We found that there was a clear degradation from PMC to uniform pin grid, and further to non-uniform pin grid, but the isolation was still good enough for non-uniform case. This was of course the best case scenario for isolation. In practice, with inclusion of bias lines and real amplifier chips, the total isolation might deteriorate. For comparison, we include in Fig. 6 (c) the computed results for a case of metal lid with and without absorbers when two adjacent dummy chips were separated by metal wall shielding. We found that the results over $\mathrm{Ka}$ band for the case of absorbers had similar levels as mentioned in [2] and were 15 20dB worse than PMC packaged cases in Fig. 6 (a).

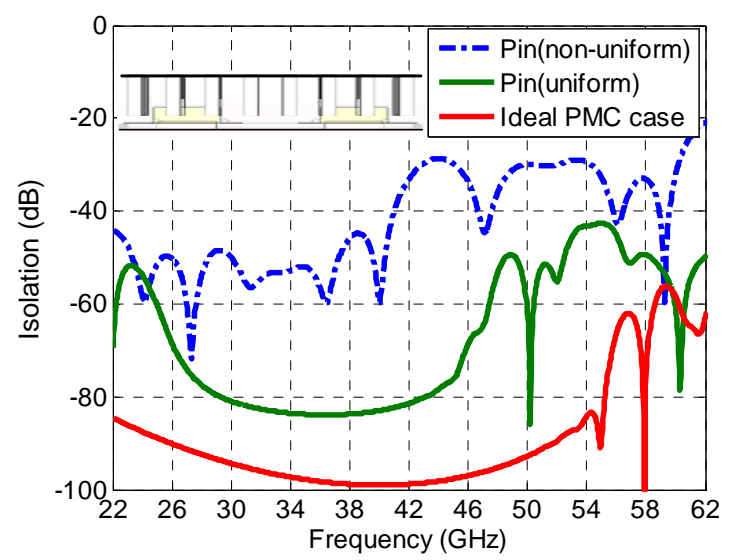

Fig.6 (a) Computed isolation between the input of one of the MMIC amplifier chip and the output of the adjacent one

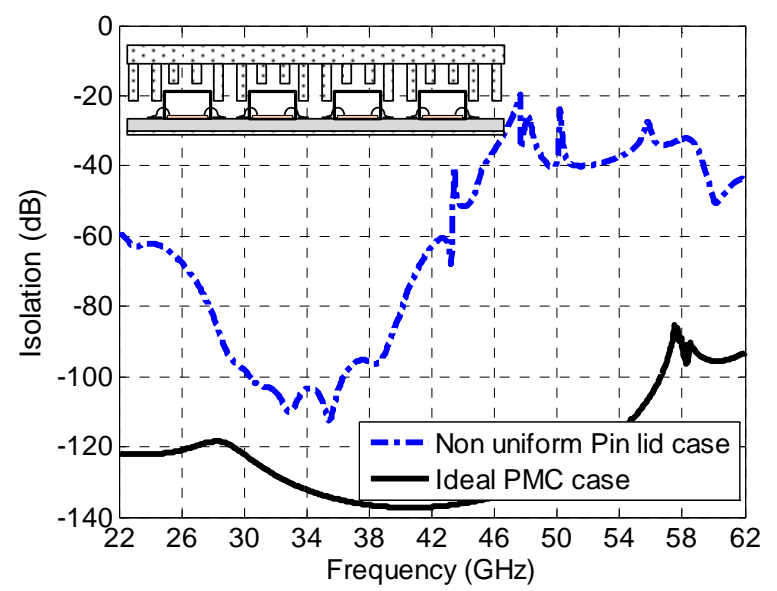

Fig.6 (b) Computed total isolation between input port and output port of the complete chain.

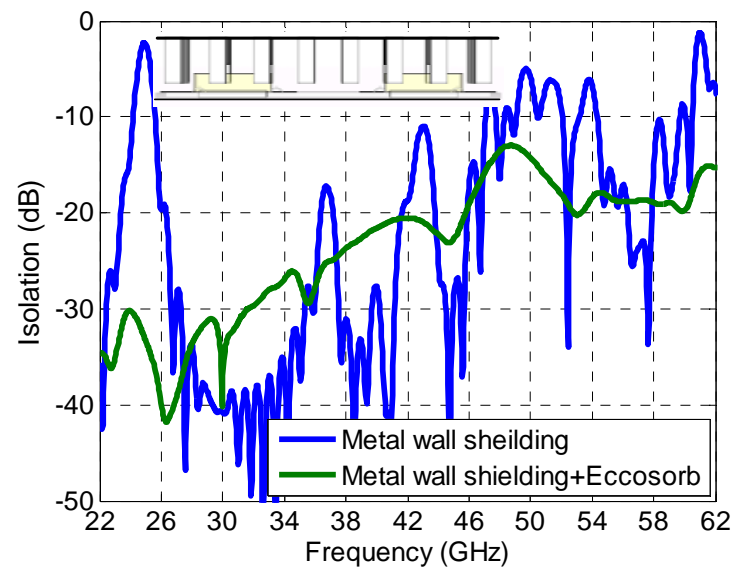

Fig.6 (c) Simulated total isolation between adjacent components with metal shielding and RF absorber. 


\section{TEST CIRCUIT FOR AMPLIFIER CHAIN ISOLATION MEASUREMENTS}

This section describes the test circuits that were used to measure the isolation between amplifier chains. The two isolation cases investigated in this work are shown below in Fig.7.
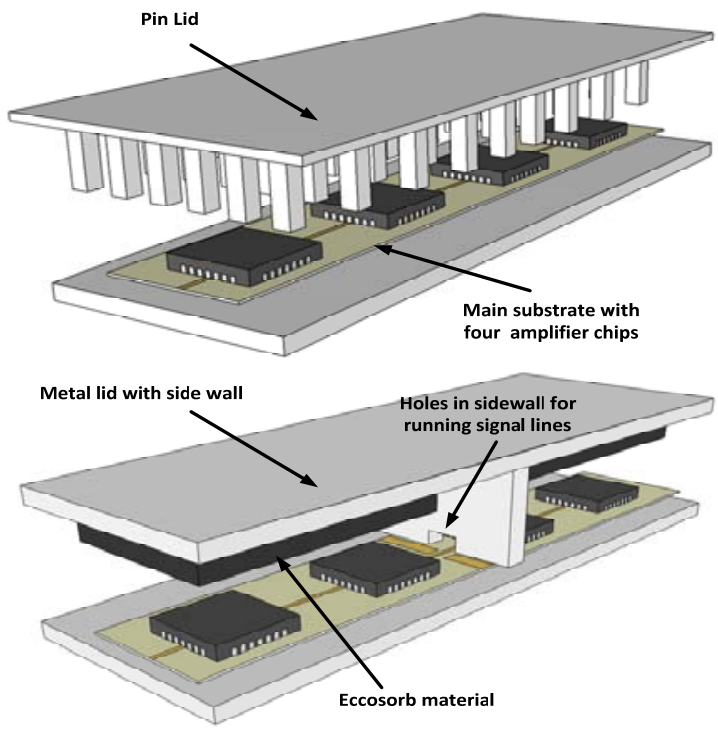

Fig.7(a) Test circuit for isolation evaluation of a single amplifier chain (side walls not shown)
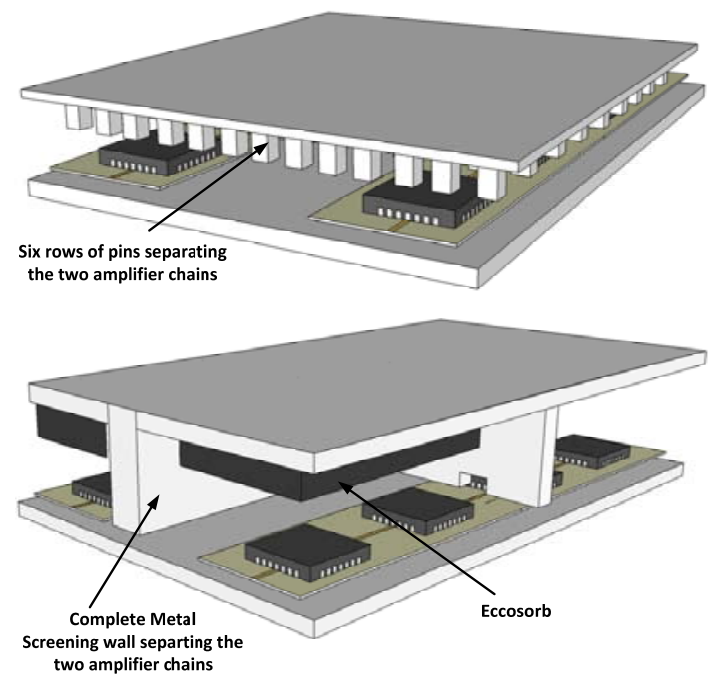

Fig.7(b) Test circuit for isolation evaluation of side by side amplifier chain (side walls not shown)

Firstly, the output to input isolation performance was tested for a single chain high gain amplifier circuit in the presence of a gap waveguide package (realized as a pin lid package) and a conventional metal wall package with absorbers. Secondly, two high gain amplifier chains were placed side by side (in opposite direction) as a model of parallel transmitter and receiver amplifier chains in a full duplex microwave system. Then, the isolation performance was evaluated for these two amplifier chains when they were shielded (packaged) with both pin lid package and a conventional complete metal wall screening or shielding.

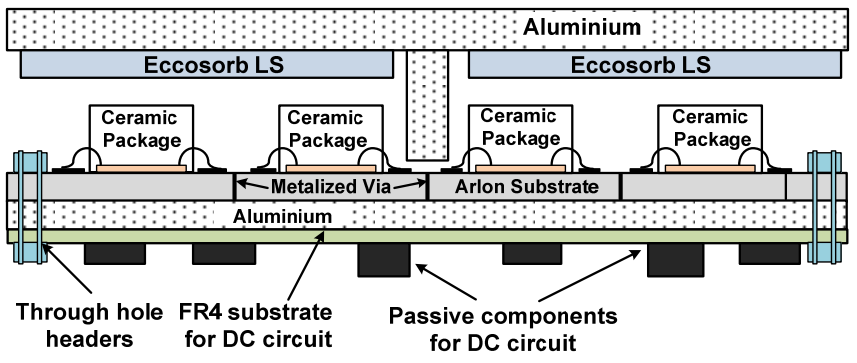

Fig.8 Test circuit with RF section and dc circuitry arrangement (side walls not shown).

The absorber used in the metal package was Eccosorb LS with a thickness of approximately $2.5 \mathrm{~mm}$, from Emerson \& Cuming. The whole structure in case of traditional metal package was divided into two sections where each cavity had a dimension of $30 \times 25 \times 4 \mathrm{~mm}^{3}$ and holds two amplifiers. This decision originated from practical design experience of having around $20 \mathrm{~dB}$ gain in each cavity. However in this work, the total possible forward gain was more than $25 \mathrm{~dB}$ in each cavity. This allowed us to perform the maximum gain test and the self-oscillation test of the amplifier chains with the necessary positive loop gain for the isolation level worse than $25 \mathrm{~dB}$. The cavity size was kept larger than the two chip sizes due to the presence of DC connectors, dc signal path and some signal lines to connect from one amplifier to the next. The dimension of the holes in the sidewalls for running the signal line from one compartment to other was $2.0 \times 1.0 \mathrm{~mm}^{2}$.

The amplifier chains used in the test circuits consisted of four variable gain amplifiers from UMS (CHA3694-QDG) and were placed on the Arlon main substrate. This main substrate was attached to a smooth metal plate by using silver epoxy. Passive components such as capacitors and resistors, as a part of the biasing network, and DC-connectors were placed in another FR4 substrate and were attached on the other side of the same metal plate. DC and RF sides were interconnected using Samtec's through-hole headers. The DC and RF arrangements are shown in Fig.8.

\section{MeAsurement Results}

\section{A. Isolation performance of single chain amplifier:}

Two experimental approaches were followed to test the isolation performance of the single chain amplifier: stable gain test and self-oscillation test, both being shown in Fig. 10. In the first approach, the amplifier chain under test was simply connected with Anritsu 36397C VNA, and a universal test fixture (UTF) from Rosenberger was used for launching the signal for S-parameter measurements. We increased the gain in the amplifier chain by varying the control voltage until there was a tendency that the amplifier started to oscillate. The UTF is an open port fixture, so we used absorber materials around the port location to absorb any possible radiation from the launching probes. In this approach, the input signal at port 1 was intentionally set to a low value in order to avoid compression in the amplifier chain. 
The second approach was a self-resonance test where the input port of the first stage amplifier was connected with a $50 \Omega$ resistor (and no signal source), and the output of the final stage amplifier was connected to a spectrum analyzer. As mentioned in the previous section, the amplifiers used in this work were variable gain amplifiers. Thus, we varied the gain of each amplifier by changing the control voltage, and observed when resonance peaks appeared at the screen of the spectrum analyzer. Both these tests were complementary to each other and were performed to cross-check the isolation performance.
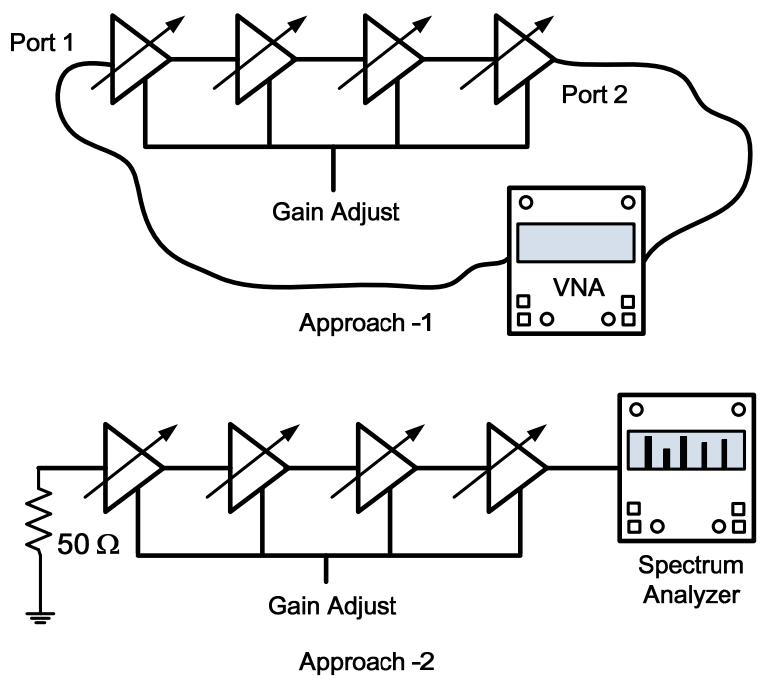

Fig.10 Test setups for single chain isolation performance test

We tested two identical amplifier chains here, one packaged with the gap waveguide pin lid and the other with a conventional metal cavity. It needs to be mentioned that the main substrate used for amplifier chain with pin lid package did not require grounded vias. On the other hand, ground strips with vias (to meet the lid walls) were used on some locations of the main PCB for amplifier chain with conventional metal wall package as shown in Fig.16. This was the reason for using two separate amplifier chains. In Fig.11, $\mathrm{S}_{21}$ measurements for a control voltage corresponding to a reduced gain are shown for these two amplifier modules. It was easily noticed from this figure that- the gain curves were not very flat over the frequency band. However, the emphasis in this work was not the matching and $\mathrm{S}_{21}$ flatness quality but on achieving the high gain in order to effectively investigate self-oscillation and feedback tendencies. Measurement results for stable gain test for a single chain amplifier is depicted in Fig. 12(a) and Fig.12 (b). It was clear that- more than 60dB stable gain was achievable for gap waveguide pin lid packaging. On the other hand, the tendency of oscillation was clearly observed already at about $40 \mathrm{~dB}$ gain in the case of a traditional metal wall package with RF absorber. Based on the small-signal s-parameters obtained during all these gain measurements, the $\mu_{1-}$ factors were calculated for the two cases of packaging under different control bias voltages and are shown in Fig. 12 (c). For pin lid package, the $\mu_{1}$ - factors remained more than 1 for both the lowest and highest bias voltages. On the other hand, for conventional metal wall packaging, the $\mu_{1}$ - factor became less than 1 at $\mathrm{V}_{\text {con }}=0.48 \mathrm{~V}$ over certain frequencies showing an unstable condition of operation.

Once the stable gain tests were performed, the selfoscillation test for the single amplifier chains was also done. As mentioned earlier, the input of the first stage amplifier in this test was connected with a $50 \Omega$ load. So, the amplifier chain would amplify only the noise signal generated within the circuit, and the noise floor would move upwards with increasing gain. The results for self-oscillation test are depicted in figures 13(a) and 13(b). From these self-oscillation measurement results, similar conclusion could be drawn as was drawn in the first approach of maximum stable gain measurements. No oscillation peaks were observed for gap waveguide packaging (pin lid packaging) even after 65 70 dB total gain. On the other hand, for traditional metal wall packaging with absorbing material, resonance peaks appeared at around $40 \mathrm{~dB}$ of total forward gain. An overall $25 \sim 30 \mathrm{~dB}$ output to input isolation improvement was thus achieved with the gap waveguide packaging technique for this high gain test amplifier chain circuit.

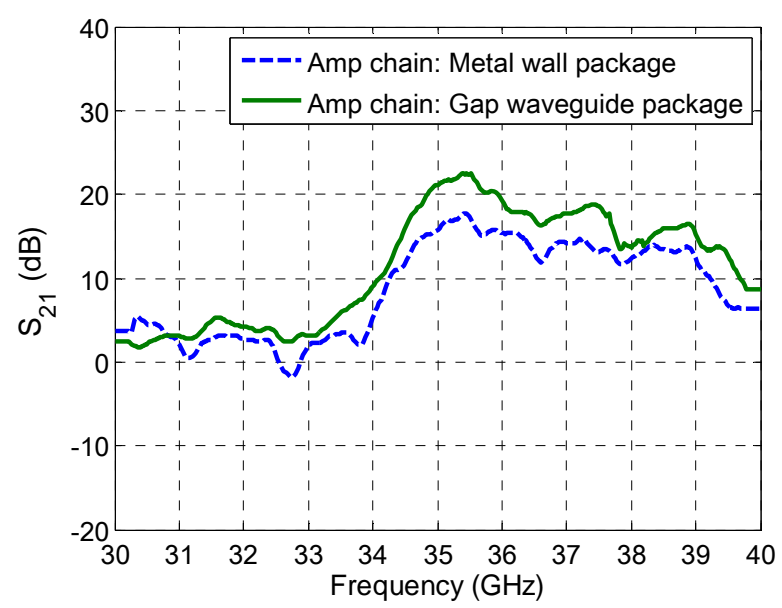

Fig.11 Measured $\mathrm{S}_{21}$ for two amplifier chains for control voltage of -0.3 volt.

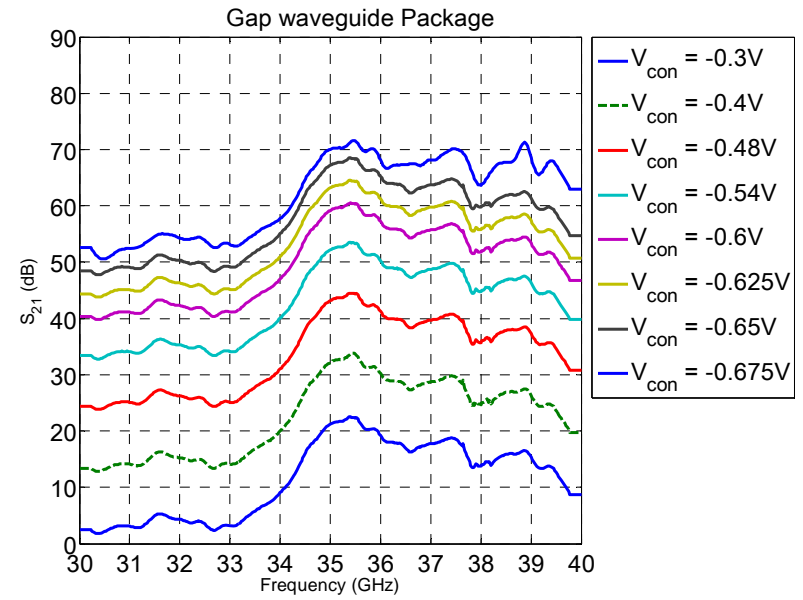

Fig.12 (a) Measured Gain curves for an amplifier chain packaged with pin lid 


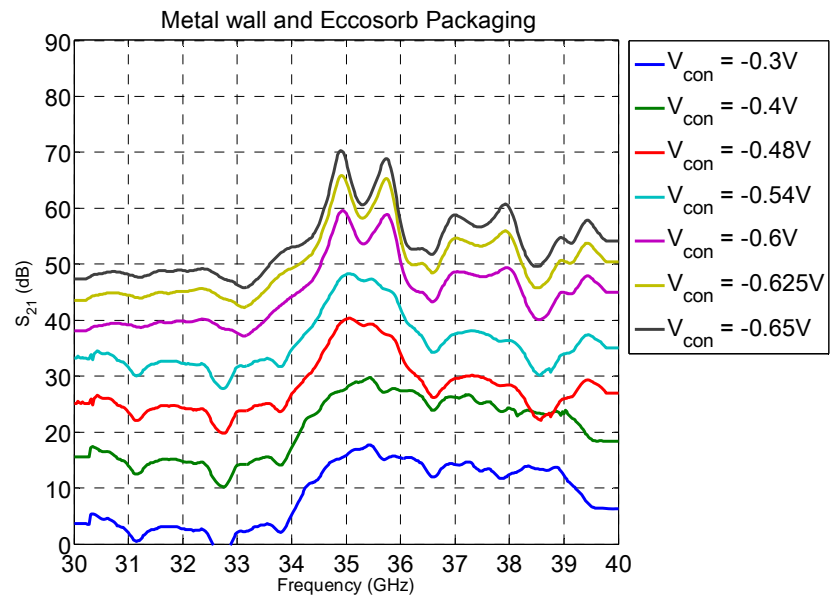

Fig.12 (b) Measured Gain curves for an amplifier chain packaged with metal wall and eccosorb

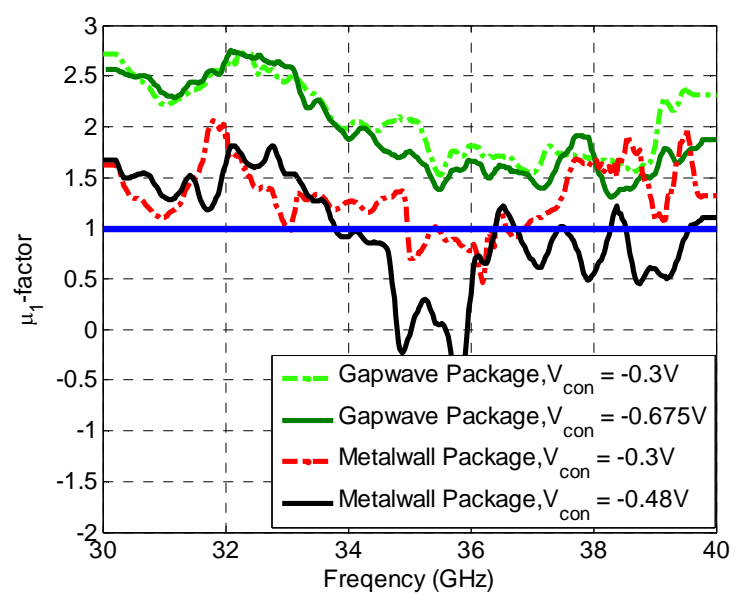

Fig. 12 (c) Calculated $\mu_{1}$ - factors for two packaging cases

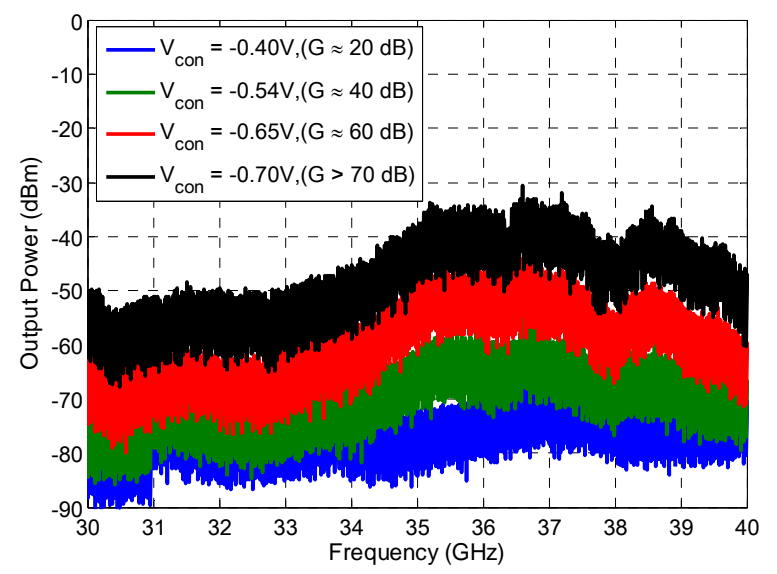

Fig.13 (a) Self-oscillation analysis for an amplifier chain packaged with pin lid.

\section{B. Isolation between two amplifier chains located side-by- side:}

All full-duplex systems with simultaneously operating transmitters and receivers require an excellent isolation between receive and transmit paths for preventing the transmit

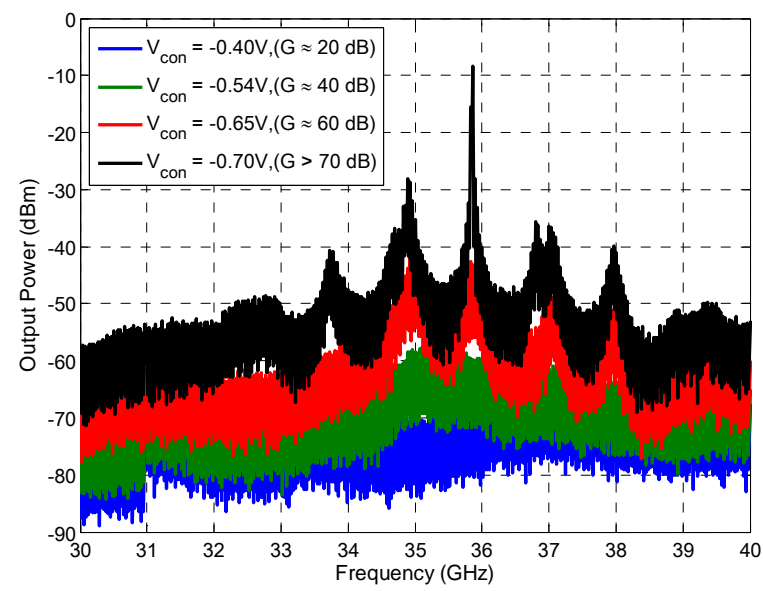

Fig.13 (b) Self-oscillation analysis for an amplifier chain packaged with metal wall and eccosorb

signal to cause saturation and intermodulation distortion in the sensitive receiver part. Particularly, the coupling between the final stage transmit amplifier and the first stage LNA of the receiver chain is of great importance due to the high difference of transmit and receive signal levels. In this work, such two amplifier chains were placed side-by-side (to emulate the transmit and receive scenario), and sidewise isolation performance was measured in presence of gap waveguide shielding and conventional metal wall shielding.

The block diagram for this test setup is shown in Fig. 14 and the details of the test circuits were already shown in Fig.7 (b). Both the amplifier chains used in the test circuit were adjusted for a moderate gain of $30 \mathrm{~dB}$ to avoid any self-oscillation and instability. The input signal was supplied from a Rohde \& Schwarz-SMR40 signal generator and the signal amplitude was always adjusted to have a constant $P_{\text {out } 1}=10 \mathrm{dBm}$ at the final stage of amplifier chain for all measurement frequency points. An Agilent-E4418BEPM power meter and Agilent8564E spectrum analyzer were used in the test setup to measure the power levels at different output stages. The measured output power at the spectrum analyzer and the isolation $I$ can be simply calculated in the following way:

$$
P_{\text {out } 1}-I+\text { Gain }=P_{\text {out } 2} ; I=P_{\text {out } 1}-P_{\text {out } 2}+\text { Gain }
$$

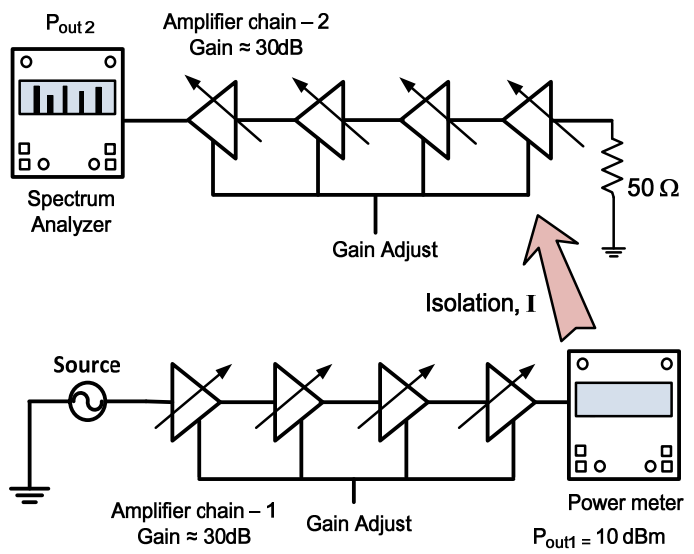

Fig.14 Test setup for measuring isolation between two amplifier chains 
It would be mentioned that two pin lid packages were used in these measurements; one having six rows of pin between the amplifier chains and the other having 8 rows of pin. The thickness of the metal wall in the conventional metal shielding was $5 \mathrm{~mm}$. The two pin lid structures are shown in Fig.15 (a) and the measured isolation for these test circuits are shown below in Fig. 15(b). In this work, we have not used any metal walls on the input and output sides of the gap waveguide packaging structures as shown in fig.16. For the other case with metal lid and absorbers, the metal walls are present on all sides. For, gap waveguide structures, there are many pin rows between the input and output ports. Usually, after 3-rows of pins, the electromagnetic field decay up to a level of $60 \mathrm{~dB}$ [26]. With many pins present in these pin lid structures, the field leakage from input to output port due to open sidewalls will be almost negligible. However, metal walls can be used if needed for mechanical support and protection, but it will not affect the electrical performance. For such an oversized gap waveguide structure, the parallel-plate modes may propagate. However, these modes may exist only outside the designed stop-band, i.e., below $26 \mathrm{GHz}$ and above $46 \mathrm{GHz}$. So, these parallel-plate modes will not affect the amplifier chain performance and the isolation performance within $30-40 \mathrm{GHz}$.

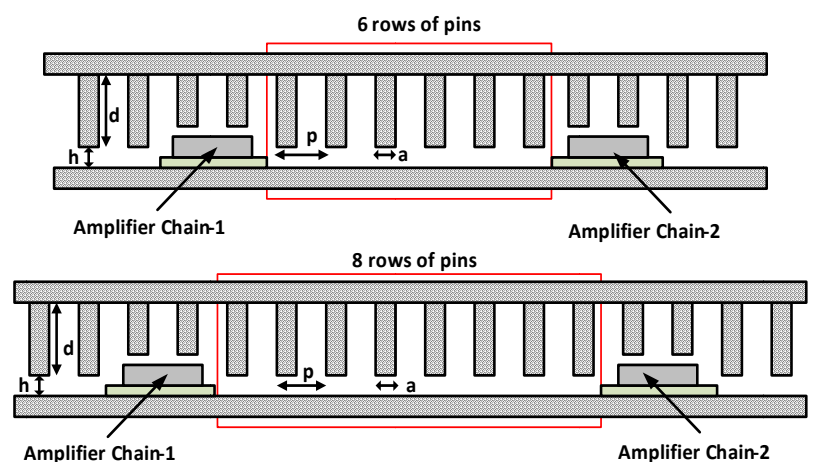

Fig.15(a) 6 row and 8 row pin lid structures; $\mathrm{a}=0.8 \mathrm{~mm}, \mathrm{~h}=0.5 \mathrm{~mm}$, $\mathrm{d}=2.25 \mathrm{~mm}$ and $\mathrm{p}=2.25 \mathrm{~mm}$.

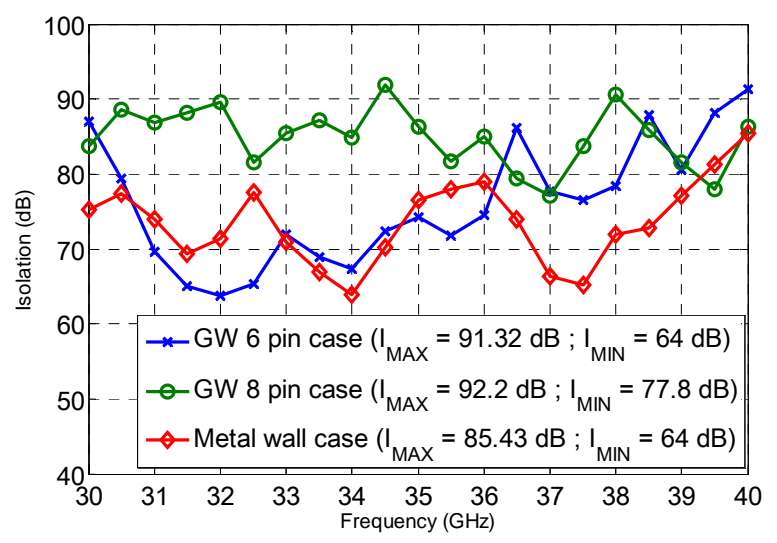

Fig.15(b) Isolation between two closely spaced amplifier chains

The measurement results showed that an isolation round $70 \mathrm{~dB}$ in average was achieved for a six pin row lid with isolation value ranging from $64 \mathrm{~dB}$ to $91 \mathrm{~dB}$. Similar isolation trend was observed also for complete metal wall shielding case. For the case of eight rows of pins, better isolation was achieved with an average isolation value of $85 \mathrm{~dB}$ and isolation values ranging from $78 \mathrm{~dB}$ to $92 \mathrm{~dB}$. These measured results have of course some errors due to the human factor when manually biasing the amplifiers in different chains, component variations in different chains etc. However, this error level was difficult to quantify at this point and was expected to be not more than $\pm 1 \sim 2 \mathrm{~dB}$. The picture of all the test circuits and the manufactured packaging lids are shown below in Fig. 16.

\section{CONCLUSION}

In this work, a new packaging or enclosure technique based on gap waveguide technology has been proposed. High isolation among various microwave components within a microwave module has been achieved without dividing the module in multiple compartments. The concept is particularly useful for active components such as high gain amplifier chains where package feedback problems, over the air coupling and coupling via substrate modes can play a critical role in cross-talk or signal integrity issue and may drive the amplifier chain towards unwanted oscillation. The new packaging technique uses an artificial magnetic conductor (AMC) on top of a circuit board. The ground plane of the circuit board and the AMC lid creates a stopband for all possible unwanted parallel-plate modes. As a result, high isolation is achieved within this stopband.
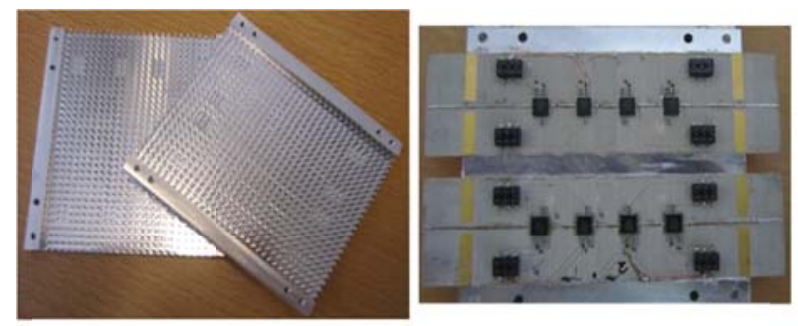

Two pin lids
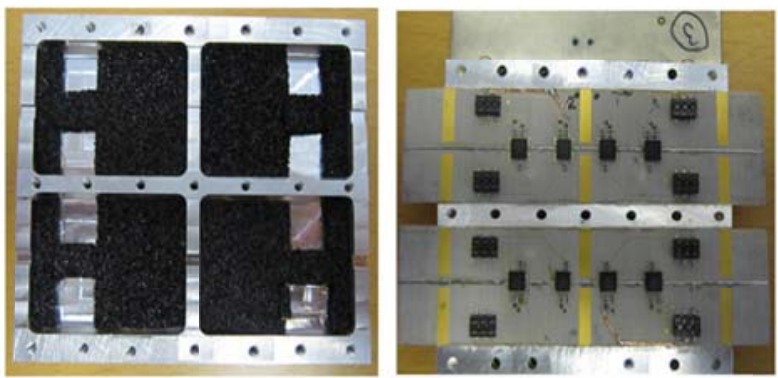

Metal wall with Eccosorb

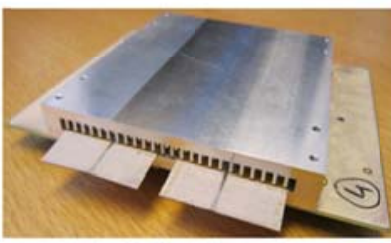

Top side ( mounted with Pin lid)

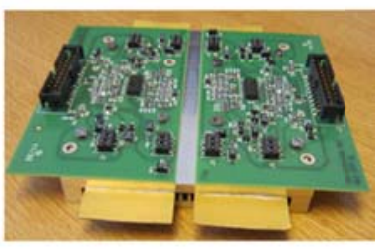

Bottom side ( Bias circuitry )
Fig.16 Pictures of all the test circuits

Throughout this work, the PMC was realized as a uniform grid of metal pins, which could be easily manufactured by a 
simple milling machine or metal sawing. The above mentioned gap waveguide package with a pin lid had been used to test the isolation performance in the case of a single amplifier chain and two side by-side-amplifier chains in 30$40 \mathrm{GHz}$ band. In the case of the single chain amplifier, the pin lid packaging offered an isolation improvement of $25 \sim 30 \mathrm{~dB}$ over the metal lid with absorber, and more than $65 \mathrm{~dB}$ of stable gain was achieved, whereas in the traditional metal wall package the oscillation tendency was clearly observed about $40 \mathrm{~dB}$ of total gain. Both the maximum stable gain test and self-oscillation test results agreed with each other and confirmed the above statement. Finally, the isolation measurements had been done for two amplifier chains placed in close proximity. Here also, the pin lid package with 8 rows of pins showed better isolation performance. A minimum isolation of about $78 \mathrm{~dB}$ had been achieved with 8 rows of pins between the two chains, whereas the traditional complete metal wall shielding provided a minimum isolation level of $64 \mathrm{~dB}$. In short, the proposed gap waveguide packaging technique is a robust and effective packaging solution for high frequency multi-compartment microwave modules and offers better isolation performance than traditional metal wall solutions with RF absorber within a specific frequency band.

\section{ACKNOWLEDGMENT}

The authors would like to thank Tomas Östling and Johan Carlsson of Arkivator AB, Göteborg, Sweden for their help in manufacturing the pin lid package and metal wall package used in measurements.

\section{REFERENCES}

[1] A.Margomenos, K.J.Herrick, M.I.Herman, S.Valas, and L. P.B.Katehi, "Isolation in Three-Dimensional Integrated Circuits," IEEE Trans. Micro. Theory and Techniques, vol. 51, no. 1, pp. 25-32, January 2003.

[2] L.Schulwitz, S.Ortiz, and A. Mortazawi, "Packaging Method for Increased Isolation Using a Mcirostrip to Waveguide Transition," IEEE Microwave and Wireless Component Letters, vol. 17, no. 3, pp. 163-165, March 2007.

[3] T.A Midford, John J. Wooldridge, and Rich L. Sturdivant, "Evolutions of Packages for Monolithic Microwave and Millimeter Wave Circuits," IEEE Transection o Antennas and Propagation, vol. 43, no. 9, pp. 983991, September 1995.

[4] M.Tsuji, H. Shigesawa, and A.A. Oliner, "Simultaneous Propagation of bound and leaky dominant modes on printed-circuit lines," IEEE Trans. Microwave Theory and Techniques, vol. 43, no. 12, pp. 3007-3019, December 1995

[5] F. Mesa, A. A. Oliner, D. R. Jackson, and M. J. Freire, "The influence of a top cover on the leakage from microstrip line," IEEE Trans. Microwave Theory and Techniques, vol. 48, no. 12, pp. 2240-2248, December 2000.

[6] W.E.McKinzie and N.G. Alexopoulos, "Leakage losses for the dominant mode of conductor-backed coplanar waveguide," IEEE Microwave Guided Wave Letter, vol. 2, pp. 65-66, February 1992.

[7] S.-J. Kim, H.-S. Yoon, and H.-Y. Lee, "Suppression of leakage resonance in coplanar MMIC packages using a Si sub-mount layer," IEEE Trans. Microwave Theory Tech., vol. 48, no. 12, pp. 2664-2669, December 2000.

[8] D. f. Williams, "Damping of the Resonant Modes of a Rectangular Metal Package," IEEE Transection on Microwave Theory and Techniques, vol. 37, no. 1, pp. 253-256, January 1989.
[9] P. Dixon, "Cavity-Resonance dampening," IEEE Microwave Magazine, vol. 6, pp. 74-84, June 2005.

[10] N. K. Das, "Methods of suppression or avoidance of parallel-plate power leakage from conductor-backed transmission lines," IEEE Trans. on Microwave Theory and Technique, vol. 44, no. 2, pp. 169-181, February 1996.

[11] W.H. Haydl, "On the Use of Vias in Conductor-Backed Coplanar Circuits," IEEE Trans. on Microwave Theory and Technique, vol. 50, no. 6, pp. 1571-1577, June 2002.

[12] G.E. Ponchak, D. Chun, J.-G. Yook and L.P.B.Katehi, "The Use of Metal filled Via Holes for Improving Isolation in LTCC RF and Wireless Multichip Packages," IEEE Trans. Advanced Packaging, vol. 23, no. 1, pp. 88-99, February 2000.

[13] S. Wane and D. Bajon, "Full-Wave analysis of Inhomogeneous Deeptrench Isolation Patterning for Substrate Coupling Reduction and QFactor Improvement," IEEE Trans. on Microwave Theory adn Technique, vol. 54, no. 12, pp. 4397-4411, December 2006.

[14] C. B. Ashesh, D. Bhattacharya and R. Garg, "Characterization of VGroove Coupled Microshield Line," IEEE Microwave and Wireless Components Letter, vol. 15, no. 2, pp. 110-112, February 2005.

[15] D.Sievenpiper, L.Zhang, R.F.J.Broas, N.G.Alexopolous, and E. Yablonovitch, "High- impedance electromagnetic surfaces with a forbidden frequency band," IEEE Trans. Microwave Theory Tech., vol. 47, no. 11, pp. 2059-2074, November 1999.

[16] R. Abhari and G. V. Eleftheriades, " Metallo-dielectric electromagnetic bandgap structures for suppression and isolation of the parallel-plate noise in high-speed circuits," IEEE Trans. Microw. Theory Tech, vol. 51, no. 6, pp. 1629-1639, June 2003.

[17] T.-Lin Wu, Y.-Hui Lin, T.-Kuang Wang, C.-C. Wang and S-T. Chen, "Electromagnetic Bandgap Power/Ground Planes for Wideband Suppression of Ground Bounce Noise and Radiated Emission in HighSpeed Circuits," IEEE Trans. Microw. Theory Tech, vol. 53, no. 9, pp. 2935-2942, September 2005.

[18] A.Uz Zaman, M.S. Ellis and P.-S. Kildal, "Metamatrial based Packaging Method for Improved Isolation of Circuit Elements in Microwave Module," accepted for publication in 42nd Eur. Microwave Conf. Amsterdam, October 2012.

[19] M.G. Silveirinha, C.A.Fernandes and J.R. Costa, "Electromagnetic characterization of textured surfaces formed by metallic pins," IEEE Trans. Antennas Propagat, vol. 56, no. 2, pp. 405-415, February 2008.

[20] P.-S. Kildal, E-Alfonso, A. Valero-Nogueira and E. Rajo-Iglesias, "Local metamaterial-based waveguides in gaps between parallel metal plates," IEEE Antennas and Wireless Propagation Letters, vol. 8, pp. 84-87, 2009.

[21] P.-S. Kildal, "Three metamaterial-based gap waveguides between parallel metal plates for $\mathrm{mm} / \mathrm{submm}$ waves," 3rd European Conf. on Antennas and Propagataion, Berlin, pp. 23-27, March 2009.

[22] P.-S. Kildal, A. Uz Zaman, E. Rajo-Iglesias, E. Alfonso and A. ValeroNogueira, "Design and experimental verification of ridge gap waveguides in bed of nails for parallel plate mode suppression," IET Microwave, Antennas \& Propagation, vol. 5, no. 3, pp. 262-270, March 2011.

[23] A. Uz. Zaman, P.-S. Kildal, M. Ferndahl and A. Kishk, "Validation of Ridge Gap Waveguide Performance Using in-house TRL Calibration Kit," 4th European Conference on Antennas and Propagation, Barcelona, April 2010.

[24] A.Valero-Nogueira, E.Alfonso, J. I. Herranz and P.-S. Kildal, "Experimental demonstration of local quasi-TEM gap modes in singlehard-wall waveguides," IEEE Microwave and Wireless Components Letters, vol. 19, no. 9, pp. 536-538, September 2009.

[25] E. Rajo-Iglesias and P.-S. Kildal, "Numerical studies of bandwidth of parallel plate cut-off realized by bed of nails, corrugations and mushroom-type EBG for use in gap wave-guides," IET Microwaves, Antennas \& Propagation, vol. 5, no. 3, pp. 282-289, March 2011.

[26] A. Uz Zaman, P.-S. Kildal, and A.Kishk, "Narrow-Band Microwave Filter Using High Q Groove Gap Waveguide Resonators with Manufacturing Flexibility and no Sidewalls," IEEE Transactions on Components, Packaging and Manufacturing Technology, vol.2,no.11, pp.1882-1888, November,2012. 
[27] A. Uz Zaman, and P.S.-Kildal, "Slot Antenna in Gap Waveguide Technology," 6th European Conference on Antennas and Propagation, EUCAP, Prague, pp. 3243-3244, March 2012..

[28] H. Raza and J.Yang, "A Low Loss Rat Race Balun in Gap Waveguide Technology," 5th European Conference on Antennas and Propagation (EUCAP), Rome, pp. 1230-1232, April 2011.

[29] H. Kirino and K. Ogawa, "A 76 GHz Multi-Layered Phased Array Antenna Using a Non-Metal Contact Metamaterial Waveguide," IEEE Transactions on Antennas and Propagation, vol. 60, no. 2, pp. 840-853, February 2012.

[30] E. Rajo-Iglesias, A. Uz Zaman,and P.-S. Kildal, "Parallel plate cavity mode suppression in microstrip circuit packages using a lid of nails," IEEE Microwave and Wireless Components Letters, vol. 20, no. 1, pp. 31-33, December 2009..

[31] A.Uz. Zaman, J.Yang, and P.-S. Kildal, "Using Lid of Pins for Packaging of Microstrip Board for Descrambling the Ports of Eleven Antenna for Radio Telescope Applications," IEEE Antennas and Propagation Society International Symposium, July 2010.

[32] A. Algaba Brazález, A. Uz Zaman, and P.-S. Kildal, "Improved Microstrip filters Using PMC Packaging by Lid of Nails," IEEE Trans. Component,Packaging and Manufacturing Technology, vol. 2, no. 7, pp. 1075-1084, July 2012.

[33] A. Kishk, A. Uz Zaman,and P.-S. Kildal, "Numerical Prepackaging with PMC lid - Efficient and Simple Design Procedure for Microstrip Circuits including the Packaging," ACES Applied Computational Society journa, vol. 27, no. 5, pp. 389-398, May 2012.

[34] S. Weinreb, P. C. Chao, and W. Copp, "Full-waveguide band, 90 to 140 GHz MMIC Amplifier module," IEEE MTT-S Int Microwave Symp., pp. 1279-1280, June 1997

[35] W. H. Haydl, "Resonance phenomena and power loss in conductorbacked coplanar structures," IEEE Microwave Guided Wave Letter, vol 20, pp. 514-516, December 2000

[36] M.A.Morgan, "Millimeter-wave MMICs and Applications," Ph.D. dissertation, California Institute of Technology, Pasadena, California, 2003.

[37] E. Rajo-Iglesias, P.-S. Kildal, and A. Kishk, "Packaging of microstrip circuits using bed of springs to suppress cavity modes - a replacement for bed of nails," IEEE MTT-S International Symp., pp. 405-408, May 2010.

[38] C.-N. Kuo, B. Houshmand, and T. Itoh, "Full-Wave Analysis of Packaged Microwave Circuits with Active and Nonlinear Devices: An FDTD Approach," IEEE Trans. Microwave Theory Tech., vol. 45, no. 5, pp. 819-826, May 1997.

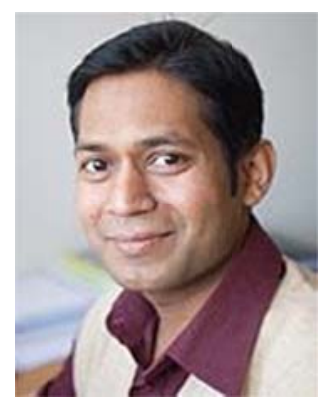

Ashraf Uz Zaman was born in Chittagong, Bangladesh. He received his BSc. in Electrical and Electrical Engineering from Chittagong University of Engineering and Technology, Bangladesh. In 2007, he received his MSc. degree from Chalmers University of Technology, Sweden. At present, he is with the Communication, Information Theory and Antenna division of same university and is working towards his $\mathrm{PhD}$. His main research interest includes millimeter waveguide technology, frequency selective surfaces, microwave passive components, packaging techniques, integration of MMIC with the antennas etc.

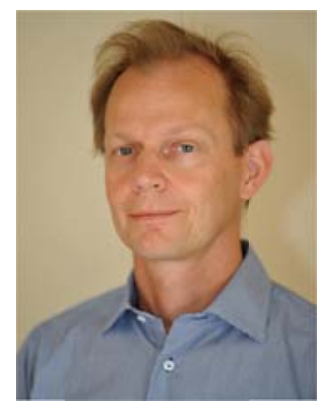

Mats Alexanderson was born in Fristad, Sweden. He received his MSc degree in Electrical Engineering in 1994 from Chalmers University of Technology, Sweden. He has since been employed as a microwave designer and specialist by companies Ericsson $\mathrm{AB}$, Viking Microwave AB, SAF Tehnika Sweden $\mathrm{AB}$ and is currently with Trebax $\mathrm{AB}$ as a consulting microwave specialist. His interests within microwave $R \& D$ include oscillator and synthesizer technology, MMIC design and technologies, active and passive circuit design, high frequency component packaging as well as microwave and millimeter wave PCB and MCM build methods.

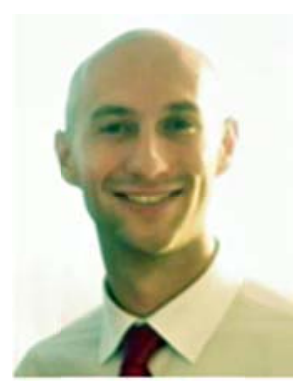

Tin Vukusic was born in Prijedor, BosniaHerzegovina. He received the M.S. degree in electrical engineering from Chalmers University of Technology, Göteborg, Sweden, in 2010. He is currently working on research and development of microwave components and modules at a consulting company TREBAX AB, Göteborg, Sweden. His interest areas within microwave R\&D include antennas for telecommunications and automotive applications, MMIC design and PCB design, and also high frequency component packaging.

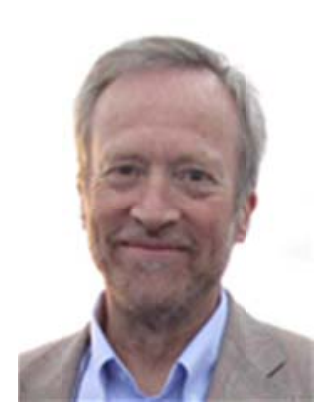

Per-Simon Kildal (M'76-SM'81-F'95) is professor in antennas at Chalmers University of Technology in Gothenburg, Sweden since 1989. $\mathrm{He}$ is heading the Antenna group. His main tasks are to lead and supervise research and education within antenna systems. Until now, 18 graduate students have received a Ph.D. from him. Kildal received himself two doctoral degrees from the Norwegian Institute of Technology in Trondheim. Kildal has authored more than 120 articles in scientific journals; concerning antenna theory, analysis, design and measurements, two of which was awarded best paper awards by IEEE (1985 R.W.P. King Award and 1991 Schelkunoff Prize Paper Award). In 2011 he received the prestigious Distinguished Achievements Award from the IEEE Antennas and Propagation Society. Kildal has done the electrical design of the $40 \mathrm{~m} \times 120 \mathrm{~m}$ cylindrical reflector antenna and line feed of the EISCAT scientific organization, and the dualreflector Gregorian feed of the $300 \mathrm{~m}$ radio telescope in Arecibo. He is the inventor behind technologies such as dipole with beam forming ring, the hat antenna, and the eleven feed. Kildal was the first to introduce the reverberation chamber as an accurate measurement instrument tool for OverThe-Air (OTA) characterization of small antennas and wireless terminals for use in multipath environments with fading. Kildal is also the originator of the concept of soft and hard surfaces from 1988, today being regarded as the first metamaterials concept. This concept is the basis of his latest and most fundamental invention, the gap waveguide technology. His research is innovative and industrially oriented, and has resulted in several patents and related spinoff companies, the most known being Bluetest $\mathrm{AB}$, see www.kildal.se for more details. Kildal organizes and lectures in courses within the European School of Antenna (ESoA, www.antennasvce.org). His textbook Foundations of Antennas - A Unified Approach (Lund, Sweden: Studentlitteratur, 2000) was well received, and is now in the process of being revised. 